\title{
Assessing the Efficacy of Chinese Herbal Medicine for Dialysis-related Myofascial Pain in the Fistula Arm: Study Protocol for a Pilot Randomized Trial
}

\section{Yung-Tang Hsu}

Chang Gung Memorial Hospital Kaohsiung Branch

Hwee-Yeong Ng

Chang Gung Memorial Hospital Kaohsiung Branch

Yan-Yuh Lee

Chang Gung Memorial Hospital Kaohsiung Branch

Yu-Chuen Huang

Chang Gung Memorial Hospital Kaohsiung Branch

Ming-Yen Tsai ( $\nabla$ missuriae@yahoo.com.tw)

Chang Gung Memorial Hospital Kaohsiung Branch https://orcid.org/0000-0001-7722-3331

\section{Study Protocol}

Keywords: Myofascial pain, Hemodialysis, Chinese herbal medicine, Randomized trial

Posted Date: June 30th, 2021

DOI: https://doi.org/10.21203/rs.3.rs-629871/v1

License: (c) (i) This work is licensed under a Creative Commons Attribution 4.0 International License. Read Full License 


\section{Abstract}

\section{Background}

Dialysis-related myofascial pain in hemodialysis (HD) patients is an important issue that is associated with many other psychosomatic problems. Effective interventions are required to alleviate pain in this group. Chinese herbal medicine (CHM) may be a potential therapeutic treatment to reduce pain. The aim of this study is to evaluate the effect of a CHM formula intervention on pain intensity, daily function, quality of life, and safety among patients receiving HD in a dialysis center within a southern Taiwan context.

\section{Methods}

This will be a randomized, open label, cross-over trial with two parallel groups in a pre- and post-test study. Forty patients with myofascial pain related to their arteriovenous fistula in the arm during regular HD sessions will be recruited. Participants will receive 4 weeks of treatment with Juan Bi Tang (JBT) and 4 weeks of no treatment in a random order, separated by a washout period of 2 weeks. Treatment doses ( $3 \mathrm{~g} \mathrm{JBT})$ will be consumed thrice daily. The primary outcome measure will be the Kidney Disease Quality of Life 36-Item Short-Form Survey. Secondary outcomes will include the Fugl-Meyer Assessment-arm, Visual Analogue Scale of pain, and grip strength. Outcomes will be collected before and after each intervention, for a total of four times per participant. The safety evaluation will focus on adverse events.

\section{Discussion}

This study will be the first to use $\mathrm{CHM}$ to treat patients receiving HD with dialysis-related myofascial pain in their fistula arm and to perform a complete assessment of the treatment, including records of quality of life, arm function and muscle power, severity of pain, and safety. The results of the study will provide convincing evidence on the use of JBT as an adjuvant treatment for dialysis-related myofascial pain.

\section{TRIAL REGISTRATION:}

Clinicaltrials.gov registry (NCT04417101) registered 30 May 2020.

\section{Introduction}

Myofascial pain syndrome (MPS) is characterized by localized pain, paresthesia, exquisite tenderness, restricted range of motion, and hypersensitivity at specific anatomic sites, which are termed taut bands with active myofascial trigger points (MTrPs) [1-3]. According to the International Association for the Study of Pain and the American Academy of Pain Medicine, the essential criteria for the diagnosis of MPS are hypersensitive spots that cause local pain, and symptoms that can be recreated by palpation [4]. In the United States, about 9 million people have MPS [5], and its prevalence in the general population is between $9 \%$ and $85 \%[4,6]$. 
It is often more serious in females than in males, and its prevalence apparently increases with age [7]. Currently, the etiology of MPS is poorly understood, but various aspects of its pathogenesis are being investigated $[7,8]$. The effects of MPS on quality of life (QOL), such as loss of work tolerance, fatigue, and weakness, are well documented [9]. However, few studies have focused on MPS in specific populations. Since one major complication of chronic kidney disease (CKD) or end stage kidney disease (ESRD) is rheumatic disorders, and since over half $(60 \%)$ of patients receiving hemodialysis (HD) develop musculoskeletal disorders [10], it is important to consider a differential diagnosis of MPS in these patients. Another form of MPS that patients receiving HD may develop is related to the fistula arm; in such cases, the symptoms are induced by maintenance HD.

Several factors, such as comorbidities, dialysis type, metabolic disorders, nutritional factors, biomechanical imbalance, and/or physio-psychological deconditioning may simultaneously contribute to the development of dialysis-related myofascial pain [11, 12]. However, the pathological mechanisms involved are not well known. Currently, the underlying pathologies are considered to be local injury from gross venipuncture or constant micro-trauma from venous pressure during each HD session in the affected arm muscle $[7,13]$. This stress can lead to inappropriate acetylcholine (Ach) activity at the endplate. This activity can cause an energy crisis that favors the release of nociceptive neurotransmitters. The altered ACh in turn triggers an active contractile phenomenon (taut bands), and the nociceptive neurotransmitters, which compensate for tissue hypoxemia, cause pain neurotransmission or sensations in the form of local pain and referred pain [14-16].

There is no definitive treatment for dialysis-related myofascial pain, and the most common treatment modality is based on condition-specific and stepwise pain management for ESRD $[17,18]$. Aspects of dialysis-related myofascial pain can be easily mitigated with oral or topical analgesics [17]. One problem with this approach to pain management is that such medications may have side effects such as bleeding, fluid retention, and cardiovascular events; thus, they are not appropriate for all patients [19]. In addition, certain sedative-hypnotic agents or muscle relaxants, which can alleviate symptoms of anxiety and/or depression, carry risks of hypotension and physical dependence [20,21].

The aforementioned limitations of conventional treatment have led to the present study, which advocates the use of Chinese herbal medicine (CHM) therapy for pain management. CHM therapy is safe and inexpensive, and it can alleviate the pain and anxiety associated with several diseases [22, 23]. Moreover, CHM used by patients with CKD have positive view to reduce the ESRD risk through analysis the prescription-related information [24].

The use of Juan Bi Tang (JBT) dates back to the middle of the thirteenth century, when it was described in the Prescriptions for Succouring the Sickness / Ji Sheng Fang, a classic Chinese medical book written by Yan Yong-He. This CHM formula is commonly used to treat Bi syndromes, such as rheumatoid arthritis, osteoarthritis, rheumatism, fibromyalgia, or myofascial syndrome, which are expressed as pain or numbness of the limbs [25]. JBT is also used for treating pain in the upper limbs, which results from the deficiency qi and blood of the collateral vessels, by warming up the meridian so as to dissipate cold, 
eliminate dampness, activate the blood, and also resolve stasis [26]. Although JBT is widely used in the treatment of musculoskeletal disorders, no studies have been conducted to examine its efficacy and safety for the treatment of dialysis-related myofascial pain in patients receiving HD. This randomized, open label, parallel-group, cross-over, single center clinical trial will aim to determine the possible benefits of JBT in patients with dialysis-related myofascial pain.

\section{Methods/design}

\section{Study design}

This single-center, two-phase study will last 12 weeks. It will be a randomized, prospective, crossover, open-label study comprising 2 groups and 2 treatment sequences, each consisting of 4 weeks of daily treatments separated by a 2-week washout period (Figure 1). In total, 40 patients will be recruited (20 patients per group) from a dialysis center in Chang Gung Memorial Hospital (CGMG), Kaohsiung, Taiwan. The subjects will be randomly and equally assigned with a central registration method into group A (JBT course in period I but not in period II) or group B (JBT course not in period I but in period II). The research protocol has been approved from the Ethics Committee of the CGMH (202000477A3) and registered at ClinicalTrials.gov (NCT04417101). The Standard Protocol Items: Recommendation for Interventional Trials (SPIRIT) 2013 checklist is provided in Additional file 1.

\section{Study participants}

Participants will be recruited from Kaohsiung CGMH by posters and advertisements on the official hospital website. The recruitment period is planned to last 12 months. All participants will provide informed consent before randomization.

\section{Eligibility criteria}

\section{Inclusion criteria}

Participants meeting the following criteria are eligible:

1. Adults (aged $\geq 20 \mathrm{y}$ ) undergoing conventional HD three times a week via an arteriovenous (AV) fistula;

2. MTrPs in one or more muscles around the AV fistula, as follows: the brachioradialis, the flexor carpi radialis, the palmaris longus, and/or the pronator teres (Figure 2), diagnosed by dialysis-related myofascial pain, which must present during HD [27];

3. Onset of symptoms within 1 month before enrollment;

4. Capability to give adequate response to pain;

5. Exquisite tenderness on palpation in the taut band, with moderate intensity of pain at baseline (i.e., a pain score $>3$ on a numeric rating scale);

6. Commitment to not changing their medication and dialysis method during the 10 weeks of this study. 


\section{Exclusion criteria}

The exclusion criteria are as follows:

1. Severe chronic or acute disease interfering with therapy attendance;

2. Comorbid conditions such as rheumatoid arthritis, stroke, tumor, coagulopathy, chronic liver disease, radiculopathies of the upper limb, recent history of cervical/shoulder/arm surgery, or trauma;

3. Depression and/or presence of a psychiatric disorder;

4. Consumption of painkillers, muscle relaxants, and anti-inflammatory medications or use of topical anesthetics in the past week;

5. Allergy to Chinese herbal products;

6. Inability to comprehend or sign an informed consent form.

\section{Subject withdrawal criteria}

Study participation will be terminated under the following conditions:

1. Occurrence of severe complications and/or general health deterioration (i.e. sepsis, bleeding, AV fistula occlusion, and progress of electrolyte disorders such as ECG change of hyperkalemia, or uncontrolled calcium $x$ phosphorus (Ca $\times$ P) level);

2. Occurrence of any severe adverse event (AE) during the intervention;

3. Voluntary withdrawal from the trial, absence from two consecutive interviews, or loss to follow-up.

\section{Interventions}

JBT Extract Granules for prescription (Product code; NO.0321131, Ko Da Pharmaceutical Co. Ltd, Taoyuan, Taiwan) will be the CHM product investigated. It is manufactured according to Good Manufacturing Practices (GMP) and adapted to factory release test. JBT consists of $4 \mathrm{~g} R x$. Angelica

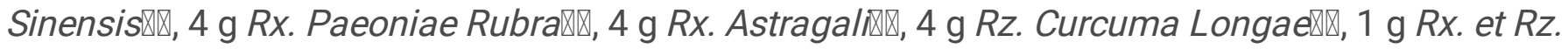

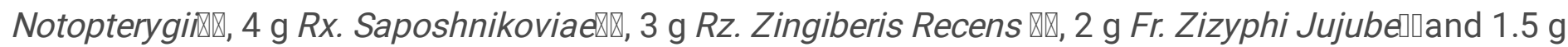
$R x$. Glycyrrhizae Preparata姆. These raw herb materials, together with starch excipient, are extracted and concentrated according to a standardized Chinese formula (see:

http://www.koda.com.tw/tqm04_e.aspx). The participants will be instructed to take JBT in doses of $3 \mathrm{~g}$ (per bag) each time, thrice daily, for 4 weeks. Participants in the non-treatment period will receive simple self-care measures for MPS based on the non-pharmacologic intervention in patients with chronic kidney disease by Pham et al. 2017, mainly including physical activity and stretching [17]. No analgesic medicines will be allowed during the intervention course. The duration of the intervention will be 8 weeks, and the follow-up will be at 2 weeks.

\section{Randomization and allocation}


The participants will be allocated into group $A(n=20)$ or group $B(n=20)$ according to a randomized list generated in Microsoft Excel 2016. The physicians will have no access to the sequence, and the investigators will distribute the drugs. All members of the research team will be explicitly instructed not to inform the participants of their allocations. The clinical outcomes assessor will be blinded to the allocations to decrease the risk of observer bias. The randomized list will be secured in a cabinet until the end of the study.

\section{Outcome measurement}

The subjects will be assessed 5 times: prior to the study, at 4 weeks (at the end of period I), at 6 weeks (at the beginning of period II), at 10 weeks (at the end of period II), and at follow-up ( 2 weeks after the trial period ends).

\section{Primary outcome}

The primary outcome used in this study will be the Kidney Disease Quality of Life 36-Item Short-Form Survey (KDQOL-36), a self-report measure developed for health-related concerns of individuals who visit dialysis facilities for treatment [28]. The KDQOL-36 is available in English and was translated into Mandarin Chinese by the RAND Corporation (see: http://www.rand.org/health/surveys_tools/kdqol.html). The instrument is composed of 12 general health items and 24 kidney-specific items. The items on general health are summarized into a Physical Component Summary (PCS) score and a Mental Component Summary (MCS) score. The 24 kidney-specific items comprise three scales: symptoms and problems, burden of kidney disease, and effects of kidney disease (12, 4, and 8 items, respectively). The raw scores are transformed into a linear range of 0 to 100, where higher scores indicate better QOL [29].

\section{Secondary outcomes}

Secondary outcome measures will include arm motor function evaluated with the Fugl-Meyer Assessment for upper extremity (FMA-UE); muscle power threshold, measured with a grip algometer; and self-reported degree of pain (Visual Analogue Scale (VAS) score).

Upper limb function will be assessed with the FMA-UE, a stroke-specific test for measuring motor impairment and recovery [30]. The FMA-UE, which is widely used to evaluate upper limb motor function in patients with stroke, is often applied in studies of pain disorders in the upper extremities [31]. The maximum score of the upper extremity scale is 66 points, divided among three components: shoulderarm (36 points), wrist-hand (24 points), and coordination (6 points). The reliability and validity of the FMA-UE are excellent, and it is sufficiently sensitive for both clinical and research applications [32]. The assessment will be performed in a standardized manner to differentiate the function levels of the fistula and non-fistula arms.

Handgrip strength will be measured with a digital handgrip dynamometer (TTM-YD, Tokyo, Japan). Two consecutive measurements of the fistula-side hand at an interval of 30 seconds will be performed, and the maximal isometric reading will be used for data analysis [33]. Hand strength is used as a simple 
metric of general muscle strength for identifying functional deficits [34]. It can also provide good resolution for localized pathologies and provides outcomes that allow better understanding of the therapeutic response of the impaired hand.

Pain perception will be recorded immediately after cannulation on a VAS, a non-graduated horizontal line of $100 \mathrm{~mm}$ where $0=$ no pain at all and $100=$ as painful as possible. The VAS and grip strength measures will be assessed immediately before the first treatment; at 4, 6, and 10 weeks after the first treatment; and at follow-up.

\section{Safety assessments}

The JBT is described in the ancient literature of traditional Chinese medicine (TCM) as relatively safe, and it did not cause major toxicity in therapeutic doses in a previous clinical study [35]. However, we will still perform a series of measures to assess the safety of the JBT throughout the entire trial due to possible herb-induced toxicity or herb-dialysis interaction [36]. All safety-related variables, including vital signs, physical examination, hematological test, biochemical test, and adverse events (AE), will be recorded in the case report at every visit. Laboratory testing of the participants' blood, electrolyte, albumin, inflammatory marker, and kidney and liver function will be monitored before the intervention, at 2-week intervals during the intervention, and again at 2 weeks after trial completion. If serious AEs related to JBT occur during the study, the investigator will provide diagnosis and treatment immediately and will report the AE to the Institutional Review Board within $24 \mathrm{~h}$ of the time of recognition. All AEs will be monitored and recorded by an independent researcher unassociated with the study until recovery of the participant.

\section{Statistical analysis}

Sample size

A statistical power analysis by $G^{*}$ Power version 3.1.9.2 for the differences in the outcome measure between both groups was conducted to determine the investigated sample size to achieve adequate power $(80 \%, a=0.05)$. For repeated measures ANOVA analysis, to achieve $80 \%$ power and a $25 \%$ effect size [36], at least 34 participants will be needed. Therefore, we plan to recruit 40 participants (20 participants for each group) to compensate for possible dropouts.

\section{Data analyses}

Statistical analyses will be conducted on the outcomes and several demographic variables. Continuous data will be presented as mean \pm SD, and categorical data will be presented as frequencies and proportions. The characteristics and clinical data of patients with and without JBT treatment will be compared with the Student's t-test for continuous variables and the chi-square test for categorical variables. Repeated measures ANOVA will be conducted to compare the subjects' conditions over time. All analyses will be performed in SPSS, version 17.0 (SPSS, Inc., Chicago, IL).

\section{Discussion}


Repeated myofascial pain in the fistula arm during HD sessions is a distressing symptom reported by patients receiving HD. Due to a lack of reporting in the past, this type of pain related to the fistula arm is rarely treated adequately to meet the needs of these patients. Uncontrolled pain in the affected muscles can degrade QOL and cause nonadherence to the recommended dialysis regimen, which can in turn increase the mortality rate due to consequent cardiovascular and pulmonary events [37,38]. JBT therapy uses an ancient herbal medicine to address these painful experiences, especially the inadequate $q i$ and blood. In a previous study, compared to western medicine, JBT was found to be safe and particularly beneficial to arthritis patients with the cold and deficiency pattern [35]. Most patients who receive HD have low physical performance and low immunity [39], and researchers have found that a feature of the TCM pattern in patients receiving HD is fundamental deficiency [40]. In pathogenesis, TCM pays attention to the circulation of qi and blood, believing that if the flow is insufficient and there is a consequent loss of body nourishment, pain will result. Thus, we propose this crossover trial to examine the effectiveness of JBT therapy in alleviating dialysis-related myofascial pain around the fistula arm in patients receiving HD.

At present, the findings of most animal studies have confirmed the benefits of JBT in improving arthritis and synovitis [41-43]. Significant reductions in the serum levels of MMP-2 and MMP-9 indicate that JBT has a role to play in preventing osteoarthritis [41]. Similar effects of JBT on synovial inflammation and bone destruction through inhibiting the pro-inflammatory cytokines have been recently reported by Wang et al. [42]. In addition, a study by Zhao et al. has demonstrated that JBT ameliorates bone destruction and reduces bone loss induced by rheumatoid arthritis [43]. Therefore, it is possible that JBT may alleviate pain by not only improving blood flow but also down-regulating the inflammatory markers. This study is designed to determine whether JBT is preferred in the treatment of dialysis-related myofascial pain, compared with non-pharmacologic methods. If this trial produces the expected results, it will provide both patients and physicians an additional option for pain control. Moreover, this trial will provide preliminary data on the effects of JBT on arm power and motor function, QOL, and safety. The results will contribute to herb repurposing for managing myofascial pain in patients receiving $\mathrm{HD}$ and will provide information useful for designing a large-scale randomized controlled trial in the future.

A few study limitations should be noted. First, this study will be an open-label study because of the difficulty of preparing a suitable placebo; the pharmaceutical technology of $\mathrm{CHM}$ powder does not yet allow that. Second, it will be a crossover trial and thus may entail possible carryover effects. To minimize this possibility, we have designed a 2-week washout period between the two groups. In the event of carryover effects, only data from the first period will be used for the analysis, for it is a randomized parallelgroup design. The current sample size was calculated on the basis of a single period of data alone; thus, the estimated size will be sufficient for specific analysis. Third, the non-treatment and JBT treatment periods will be only 4 weeks.

\section{Abbreviations}

Ach 
acetylcholine; AE:adverse event; AV:arteriovenous; CGMH:Chang Gung Memorial Hospital; CHM:Chinese herbal medicine; CKD:chronic kidney disease; ESRD:end stage kidney disease; FMA-UE:Fugl-Meyer Assessment for upper extremity; GMP:Good Manufacturing Practices; JBT:Juan Bi Tang; KDQOL36:Kidney Disease Quality of Life 36-Item Short-Form Survey; MPS:myofascial pain syndrome; MTrPs:myofascial trigger points; HD:hemodialysis; QOL:quality of life; TCM:traditional Chinese medicine; VAS:visual analog scale.

\section{Declarations}

\section{Ethics approval and consent to participate}

This study protocol with the written informed consent forms has been peer-reviewed and approved by the Institutional Review Board at Chang Gung Memorial Hospital (approval No. 202000477A3) with respect to the scientific content and ethical compliance with regulations, i.e., Good Clinical Practice and relevant laws by Data and safety Monitoring Plan. A confirming informed consent will be obtained from all study participants

\section{Consent for publication}

Not applicable.

\section{Availability of data and materials}

The datasets used and/or analyzed during the current study are available from the corresponding author on reasonable request.

\section{Competing interests}

The authors declare that they have no competing interests.

\section{Funding}

This study was funded by Chang Gung Memorial Hospital with grant number CORPG-8K0091. The funders have had no role in study design and will not have any role in the trial design, manuscript writing, or decision making for publication.

\section{Authors' contributions}

MYT and YYL devised the study question and design. MYT developed the idea into the full protocol and wrote the article draft. HYN reviewed the protocol. YCH calculated the sample size and specified the statistical strategy. MYT and YTH will be in charge of enrolling participants and conducting all the procedures. All authors have read and approved the final manuscript.

\section{Acknowledgements}


The authors would like to acknowledge all physicians and nurses in the Dialysis Units of the Physical Building of Kaohsiung Chang Gung Memorial Hospital for their kind help in the recruitment and assessment of participants, and to thank Yuan's General Hospital and Dr. Yi-Ju Chen for the professional guidance in dialysis-related myofascial pain.

\section{Trial status}

The final protocol version is 1.0, dated 02 June 2020. Patient recruitment will begin in July 2020 after we acquire ethical approval, and it will be ongoing. We anticipate the recruitment phase to be completed by June 2021.

\section{References}

1. Cummings M, Baldry P. Regional myofascial pain: Diagnosis and management. Best practice research Clinical rheumatology. 2007;21:367-87.

2. Lavelle ED, Lavelle W, Smith HS. Myofascial Trigger Points. Anesthesiology Clinics. 2007;25(4):84151.

3. Fernández-de-Las-Peñas C, Dommerholt J. International Consensus on Diagnostic Criteria and Clinical Considerations of Myofascial Trigger Points: A Delphi Study. Pain medicine (Malden Mass). 2018;19(1):142-50.

4. Rivers WE, Garrigues D, Graciosa J, Harden RN. Signs and Symptoms of Myofascial Pain: An International Survey of Pain Management Providers and Proposed Preliminary Set of Diagnostic Criteria. Pain medicine (Malden Mass). 2015;16(9):1794-805.

5. Chen Q, Bensamoun S, Basford JR, Thompson JM, An KN. Identification and quantification of myofascial taut bands with magnetic resonance elastography. Arch Phys Med Rehabil. 2007;88(12):1658-61.

6. Fleckenstein J, Zaps D, Rüger LJ, Lehmeyer L, Freiberg F, Lang PM, Irnich D. Discrepancy between prevalence and perceived effectiveness of treatment methods in myofascial pain syndrome: results of a cross-sectional, nationwide survey. BMC Musculoskelet Disord. 2010;11:32.

7. Bourgaize S, Newton G, Kumbhare D, Srbely J. A comparison of the clinical manifestation and pathophysiology of myofascial pain syndrome and fibromyalgia: implications for differential diagnosis and management. J Can Chiropr Assoc. 2018;62(1):26-41.

8. Gerwin RD. Myofascial Trigger Point Pain Syndromes. Seminars in neurology. 2016;36(5):469-73.

9. Henriksson KG, Bäckman E, Henriksson C, De Laval JH. Chronic Regional Muscular Pain in Women with Precise Manipulation Work. Scand J Rheumatol. 1996;25(4):213-23.

10. Bardin T. Musculoskeletal manifestations of chronic renal failure. Curr Opin Rheumatol. 2003;15:4854.

11. Unalan H, Majlesi J, Aydin FY, Palamar D. Comparison of high-power pain threshold ultrasound therapy with local injection in the treatment of active myofascial trigger points of the upper trapezius 
muscle. Arch Phys Med Rehabil. 2011;92(4):657-62.

12. Fleishman TT, Dreiher J, Shvartzman P. Pain in Maintenance Hemodialysis Patients: A Multicenter Study. J Pain Symptom Manage. 2018;56(2):178-84.

13. Wyllie AH, Kerr JFR, Currie AR: Cell Death: The Significance of Apoptosis. In: International Review of Cytology. Volume 68, edn. Edited by Bourne GH, Danielli JF, Jeon KW: Academic Press; 1980: 251306.

14. Pal US, Kumar L, Mehta G, Singh N, Singh G, Singh M, Yadav HK. Trends in management of myofacial pain. Natl J Maxillofac Surg. 2014;5(2):109-16.

15. Hong C-Z, Simons DG. Pathophysiologic and electrophysiologic mechanisms of myofascial trigger points. Arch Phys Med Rehabil. 1998;79(7):863-72.

16. Jafri MS. Mechanisms of Myofascial Pain. Int Sch Res Notices. 2014;2014:523924.

17. Pham PC, Khaing K, Sievers TM, Pham PM, Miller JM, Pham SV, Pham PA, Pham PT. 2017 update on pain management in patients with chronic kidney disease. Clin Kidney J. 2017;10(5):688-97.

18. Santoro D, Satta E, Messina S, Costantino G, Savica V, Bellinghieri G. Pain in end-stage renal disease: a frequent and neglected clinical problem. Clin Nephrol. 2013;79(Suppl 1):2-11.

19. Hörl WH. Nonsteroidal Anti-Inflammatory Drugs and the Kidney. Pharmaceuticals (Basel Switzerland). 2010;3(7):2291-321.

20. Zheng C, Xu J, Chen C, Lin F, Shao R, Lin Z, Liu Y, Zhou Y. Effects of sleep disorders and sedativehypnotic medications on health-related quality of life in dialysis patients. Int Urol Nephrol. 2019;51(1):163-74.

21. Mina D, Johansen KL, McCulloch CE, Steinman MA, Grimes BA, Ishida JH. Muscle Relaxant Use Among Hemodialysis Patients: Prevalence, Clinical Indications, and Adverse Outcomes. American journal of kidney diseases: the official journal of the National Kidney Foundation. 2019;73(4):52532.

22. Sarris J, Panossian A, Schweitzer I, Stough C, Scholey A. Herbal medicine for depression, anxiety and insomnia: a review of psychopharmacology and clinical evidence. European neuropsychopharmacology: the journal of the European College of Neuropsychopharmacology. 2011;21(12):841-60.

23. Mist S, Wright C, Jones KD, Carson JW, Shih J. Traditional Chinese Medicine for Fibromyalgia. Practical pain management 2010, 10(7).

24. Lin MY, Chiu YW, Chang JS, Lin HL, Lee CT, Chiu GF, Kuo MC, Wu MT, Chen HC, Hwang SJ. Association of prescribed Chinese herbal medicine use with risk of end-stage renal disease in patients with chronic kidney disease. Kidney international. 2015;88(6):1365-73.

25. Zhang EQ. Bi Syndrome (Arthralgia Syndrome). Journal of traditional Chinese medicine = Chung i tsa chih ying wen pan. 2010;30(2):145-52.

26. Zhang GG, Lee WL, Lao L, Bausell B, Berman B, Handwerger B. The variability of TCM pattern diagnosis and herbal prescription on rheumatoid arthritis patients. Altern Ther Health Med. 
2004;10(1):58-63.

27. Kuan TS. Current studies on myofascial pain syndrome. Curr Pain Headache Rep. 2009;13(5):365-9.

28. Hays RD, Kallich JD, Mapes DL, Coons SJ, Carter WB. Development of the kidney disease quality of life (KDQOL) instrument. Quality of life research: an international journal of quality of life aspects of treatment care rehabilitation. 1994;3(5):329-38.

29. Lacson E Jr, Xu J, Lin SF, Dean SG, Lazarus JM, Hakim RM. A comparison of SF-36 and SF-12 composite scores and subsequent hospitalization and mortality risks in long-term dialysis patients. Clinical journal of the American Society of Nephrology: CJASN. 2010;5(2):252-60.

30. Fugl-Meyer AR, Jääskö L, Leyman I, Olsson S, Steglind S. The post-stroke hemiplegic patient. 1. a method for evaluation of physical performance. Scand J Rehabil Med. 1975;7(1):13-31.

31. Kowalczewski J, Ravid E, Prochazka A: Fully-automated test of upper-extremity function. Conference proceedings: Annual International Conference of the IEEE Engineering in Medicine and Biology Society IEEE Engineering in Medicine and Biology Society Annual Conference 2011, 2011:73327335.

32. Lin JH, Hsueh IP, Sheu CF, Hsieh CL. Psychometric properties of the sensory scale of the Fugl-Meyer Assessment in stroke patients. Clinical rehabilitation. 2004;18(4):391-7.

33. Charlson ME, Pompei P, Ales KL, MacKenzie CR. A new method of classifying prognostic comorbidity in longitudinal studies: development and validation. Journal of chronic diseases. 1987;40(5):37383.

34. Fukuda DH, Smith-Ryan AE, Kendall KL, Moon JR, Stout JR. Simplified method of clinical phenotyping for older men and women using established field-based measures. Experimental gerontology. 2013;48(12):1479-88.

35. He Y, Lu A, Zha Y, Tsang I. Differential effect on symptoms treated with traditional Chinese medicine and western combination therapy in RA patients. Complement Ther Med. 2008;16(4):206-11.

36. Zhong Y, Deng Y, Chen Y, Chuang PY, Cijiang He J. Therapeutic use of traditional Chinese herbal medications for chronic kidney diseases. Kidney international. 2013;84(6):1108-18.

37. Claxton RN, Blackhall L, Weisbord SD, Holley JL. Undertreatment of symptoms in patients on maintenance hemodialysis. J Pain Symptom Manage. 2010;39(2):211-8.

38. Madeiro AC, Machado PDLC, Bonfim IM, Braqueais AR. FET L: Adherence of chronic renal insufficiency patients to hemodialysis. Acta paul enferm. 2010;23:546-51.

39. Tsai MY, Wu CH, Huang YC, Chen SY, Ng HY, Su YJ, Chen YH. Treatment of intradialytic hypotension with an herbal acupoint therapy in hemodialysis patients: A randomized pilot study. Complement Ther Med. 2018;38:67-73.

40. Chen CS, Chen GW, Lin JK, Peng WH, MC X. Studies on the classical differential diagnosis of Traditional Chinese Medicine and biochemical parameters in hemodialysis patients. J Chin Med. 2007;18:169-78. 
41. Yuan PW, Yu HC, Zhou HZ, Zhu C, Qu Q, Liu DY. Preventive administration of juanbi capsules for knee osteoarthritis: effects on serum MMP-2 and MMP-9 levels and cartilage repair. Journal of traditional Chinese medicine $=$ Chung $\mathrm{i}$ tsa chih ying wen pan. 2011;31(4):334-7.

42. Wang T, Jia Q, Chen T, Yin H, Tian X, Lin X, Liu Y, Zhao Y, Wang Y, Shi Q, et al. Alleviation of Synovial Inflammation of Juanbi-Tang on Collagen-Induced Arthritis and TNF-Tg Mice Model. Front Pharmacol. 2020;11:45.

43. Zhao H, Xu H, Zuo Z, Wang G, Liu M, Guo M, Xiao C. Yi Shen Juan Bi Pill Ameliorates Bone Loss and Destruction Induced by Arthritis Through Modulating the Balance of Cytokines Released by Different Subpopulations of T Cells. Front Pharmacol. 2018;9:262.

\section{Tables}


Table 1

Summary of collected data at each time point according to SPIRIT 2013 guidelines

\begin{tabular}{|c|c|c|c|c|c|c|}
\hline \multirow[t]{2}{*}{ Testing variables } & \multirow{2}{*}{$\begin{array}{l}\text { Screening } \\
\text {-week } 1\end{array}$} & \multicolumn{4}{|c|}{ Randomization } & \multirow{2}{*}{$\begin{array}{l}\text { Follow- } \\
\text { up } \\
\text { Week } 12\end{array}$} \\
\hline & & $\begin{array}{l}\text { Week } 0 \\
\text { (baseline) }\end{array}$ & $\begin{array}{l}\text { Week } \\
4\end{array}$ & $\begin{array}{l}\text { Week } \\
6\end{array}$ & $\begin{array}{l}\text { Week } \\
10\end{array}$ & \\
\hline $\begin{array}{l}\text { Inclusion and exclusion } \\
\text { criteria }\end{array}$ & $x$ & & & & & \\
\hline Baseline information & $\mathrm{x}$ & & & & & \\
\hline Inform consent & $\mathrm{x}$ & & & & & \\
\hline \multicolumn{7}{|l|}{ Intervention } \\
\hline Period I & & $\mathrm{x}$ & $x$ & & & \\
\hline Period II & & & & $x$ & $x$ & \\
\hline \multicolumn{7}{|l|}{ Assessment } \\
\hline VAS & & $x$ & $x$ & $x$ & $x$ & $x$ \\
\hline KDQOL-36 & & $x$ & $x$ & $x$ & $x$ & $x$ \\
\hline FMA-UE & & $x$ & $x$ & $x$ & $x$ & $x$ \\
\hline Griping force & & $x$ & $\mathrm{x}$ & $x$ & $x$ & $x$ \\
\hline Laboratory data & & $x$ & $\mathrm{x}$ & $x$ & $x$ & $x$ \\
\hline Conditional information & & & $\mathrm{x}$ & $x$ & $x$ & \\
\hline Adverse events & & & $x$ & $x$ & $x$ & \\
\hline
\end{tabular}

\section{Figures}




\section{Enrollment}

Assessed for eligibility

\begin{tabular}{|c|c|}
\hline Randocation & Randomized ( $\mathrm{n}=40)$ \\
\hline Crossover & Juan Bi Tang intervention ( $\mathrm{n}=20$ ) \\
\hline Crossed-over to receive other period ( $\mathrm{n}=40)$ \\
\hline \\
\hline
\end{tabular}

Figure 1

Study design from enrollment to analysis following the CONSORT 2010 flow diagram 


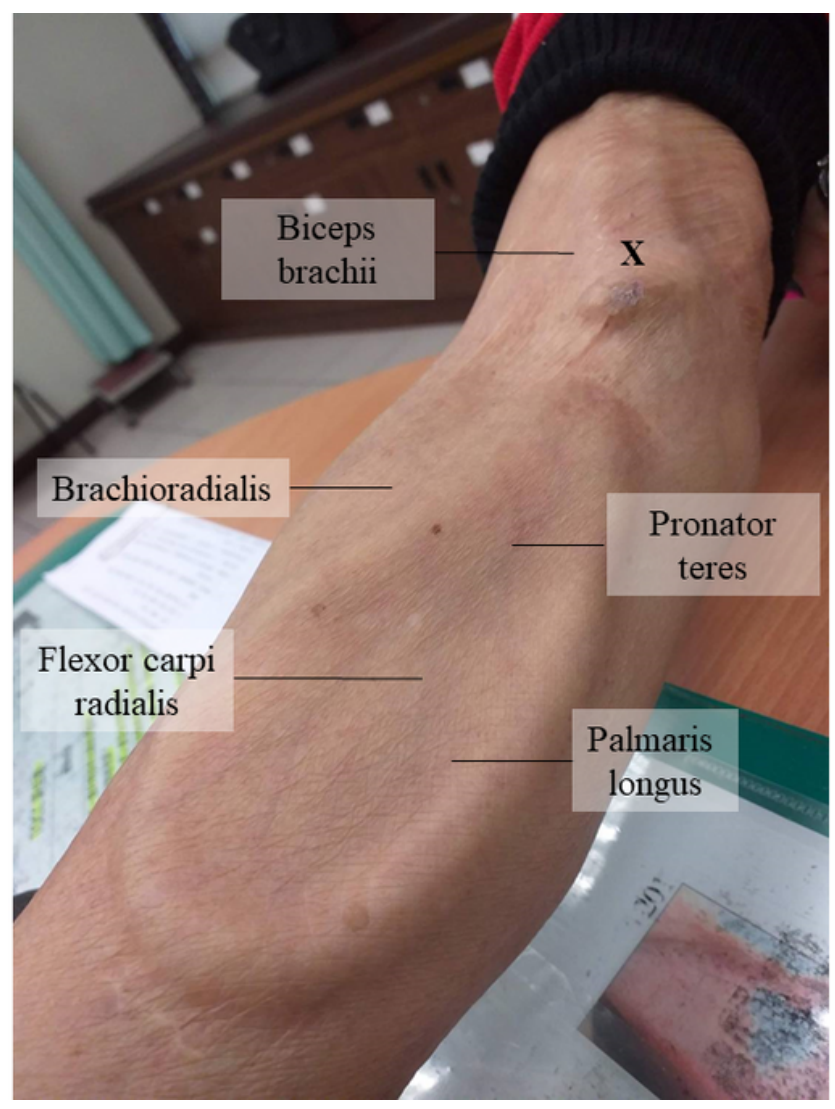

Figure 2

Muscles of the anterior forearm around the arteriovenous fistula (x: trigger points of the biceps brachii)

\section{Supplementary Files}

This is a list of supplementary files associated with this preprint. Click to download.

- 2013SPIRITFillablechecklist.pdf 